\title{
EDAD Y CRECIMIENTO DE LA BOGA DE RIO (CHONDROSTOMA POLYLEPIS, STEINDACHNER, 1865) $E N$ LA CUENCA DEL DUERO
}

\author{
E. Barceló Culebras, D. García de Jalón \\ Dpto. de Zoología y Entomología, Escuela Técnica Superior de Ingenieros de Montes, U.P.M. Madrid.
}

Palabras clave: Ch. polylepsis Age, Growth, Length-weight relationships, morphic coefficient, fish ecology.

\begin{abstract}
The populations of the lberian nase (Chondrostoma polylepsis Steind., 1865) in the Duero Basin (North of Spain) were studied. Morphic and Growth parameters were analized from Weight/Length ${ }^{3}$ ratios and from von Rertalanffy's model: a' $\left(1,4110^{-5}-1,20\right.$ $\left.10^{-5} \mathrm{gr} . / \mathrm{mm} .^{3}\right), \mathrm{k}\left(0,03-0,26 \mathrm{yr}^{-1}\right)$ and $\mathrm{L}^{\infty}(309,22-1.223,46 \mathrm{~mm}$.).

The abiotic factors that controls growth in these nase population are also studied, being the mean annual water-temperature the main one, and the water-nutrients and water-rnineralization secondarily. A mild water pollution has a negative on the lberian nase growths.
\end{abstract}

\section{INTRODUCCION}

Las poblaciones de boga (Chondrostoma polylepis Steind., 1865), especie endémica de la Península Ibérica, han sido estudiadas con relación a su biología y ecologia por diversos autores (Perez, 1979; LobonCervia y Elvira, 1981; Lobon-Cervia, 1982; Granado y García-Novo, 1983).

El presente trabajo aporta datos de los parametros mórficos y de crecimiento de poblaciones de boga pertenecientes a la Cuenca del Duero, en la que se halla ampliamente distribuida, apareciendo desde cotas de 190 m., hasta $1.200 \mathrm{~m}$., de altitud (García de Jalón y López Alvarez, 1981). Estos resultados fueron comparados a su vez con algunos de los trabajos citados anteriormente.

\section{MATERIALES Y METODOS}

Durante el otoño de 1981 y la primavera de 1982 fueron muestreadas alrededor de 70 estaciones ubicadas en la red hidrográfica de la cuenca del Duero, distribuidas de manera homogénea por toda la zona y evitando los tramos muy contaminados, estudiándose las poblaciones piscícolas y macrobénticas (García de Jalón y Gonzalez del Tánago, 1983), y analizando las aguas (Gonzalez del Tanago y Garcia de Jalón, 1982).

Limnética 2: 235-240(1986)

O Asociación Española de Limnología, Madrid. Spain
Se estudiaron un total de 154 ejemplares de boga de las que se capturaron en número suficiente, $\mathrm{N}>$ 7 , para ser analizadas separadamente, fueron tomadas de los tramos de los ríos siguientes: Duero alto (Du-a) en la provincia de Soria, Duero bajo (Du-b) en Valladolid y Zamora, Orbigo bajo (Or-b) hasta Benavides, Arlanza (Aa) en Torrepadre (Burgos), Esgueva (Eg) en Burgos, Carrión (Ca) en Villoldo (Palencia), Esla (El) desde Villaroañe (León) hasta Bretó (Zamora) y Pisuerga (Pi) en Cordovilla la Real (Palencia).

Los ejemplares de bogas capturados fueron pesados y medidos; el peso del especimen sin eviscerar se tomó con una precisión de 0,5 gr.; por otro lado fue tomada la longitud a la bifurcación caudal (Lm)(Laevastu, 1980) que va desde el hocico del pez hasta el borde de la aleta caudal en su bifurcación. Lm fue medida en $\mathrm{mm}$. con una precisión de $1 \mathrm{~mm}$.

Para la determinación de la edad fue utilizada la lectura de annuli en escamas. Las escamas se obtuvieron del flanco derecho del pez, de la zona comprendida entre la inserción de la aleta dorsal y la línea lateral, y por encima de ésta. Las escamas se conservaron en seco y se leyeron remojadas en alcohol, limpias de adherencias de grasa y mucosidad.

Los annuli aparecen a finales de mayo y principio de junio; cabe destacar que en especies de reproducción estival. como ocurre en este caso, el primer ani- 


\section{RIO}

Cuenca del Duero

Duero Alto

Duero Bajo

Pisuerga

Arlanza

Esgueva

Carrión

Esla

Orbigo Bajo

(1) Río Esla

- montaña

- meseta

(2)E. de Pinilla

- machos

- hembras

(3) Río Jarama

- machos

- hembras
$\mathrm{L}$ (mm.)

589,56

309,22

328,53

334,15

374,18

363,61

631,43

$1.151,54$

$1.223,46$

445,10

543.86

322,23

358,75

222,10

272,70
$\mathrm{K}$

0,08

0,23

0,18

0,16

0,26

0,06

0,03

0,03

$\mathrm{t}_{0}$ (años)

$-0.35$

0,57

0,65

0,26

$-0,07$

1,63

$-0,56$

$-1,16$

$-0,76$

$0,11 \quad-0,31$

0,11

$-0,23$

46,74

58,19

0,24

0,22

0,17

0,16

64,00

63,00

0,19

0,15

$-0,06$

$-0,14$

43,98

40,54

Tabla 1.-Valores de los parámetros de las ecuaciones de crecimiento lineal de Von Bertalanffy de poblaciones de ( $h$. pollkpis de la cuenca del Duero y de vanos nos. (1)datosde Pérez: (2) datos de Lobon-Cervia y Elvira (1981) y (3)datos de Lobon-Cervia (1982).

Growth parameters of Von Bertalanffy's model for C $h$. pol $\%$ pis in Duero Basin.

\begin{tabular}{|c|c|c|c|c|c|c|c|c|c|c|c|c|c|c|c|c|}
\hline RIO & $\mathrm{L}_{1}$ & $L_{i}$ & $L_{2}$ & $L_{2}^{\prime}$ & $L_{3}$ & $L_{3}$ & $\mathrm{~L}_{4}$ & $L_{4}^{-}$ & $L_{5}$ & $L_{5}^{\prime}$ & $\mathrm{L}_{6}$ & $L_{\sigma}^{\prime}$ & $L_{7}$ & $L_{7}^{\prime}$ & $L_{8}$ & $L_{8}^{\prime}$ \\
\hline Duero Al to & 64,7 & & 93,1 & 89,6 & 138,2 & 136,4 & 167,7 & 173,3 & 192,4 & 202,3 & & & \multirow{8}{*}{$\begin{array}{l}259,8 \\
260,1 \\
268,2\end{array}$} & \multirow{8}{*}{$\begin{array}{l}273,9 \\
251,9 \\
248,5\end{array}$} & \multirow{8}{*}{291,2} & \multirow{8}{*}{$\begin{array}{l}294,5 \\
275,0\end{array}$} \\
\hline Duero Bajo & 64,3 & & $\begin{array}{ll}9 & 3\end{array}$ & 7 & 131,6 & 136.7 & 171,0 & 175,9 & 202,9 & 207.0 & & & & & & \\
\hline Pisuerga & 72,4 & & 96,7 & 90,5 & 135,8 & 130.9 & 163,8 & 164,6 & 208,7 & 192,7 & 224,8 & 216,2 & & & & \\
\hline Arlanza & 61,7 & & 91,4 & 103 & 128,6 & 142,3 & 171,1 & 175,8 & 197,7 & 204,4 & 237,1 & 227,6 & & & & \\
\hline Esqueva & 99,0 & & 123,4 & & 154,6 & & 182,4 & 167,5 & 212,0 & 212,5 & 238,5 & 247,2 & & & & \\
\hline \multirow[t]{2}{*}{ Carrión } & 67,3 & 63,1 & 99,2 & 100 & 134,2 & 134,7 & 182,0 & 167,0 & $209, t$ & 197,2 & 231,0 & 225,5 & & & & \\
\hline & 52,4 & 71,7 & 83,8 & 103 & 122,2 & 134,2 & 162,3 & 164,1 & 194,7 & 193,1 & 246,1 & 221,2 & & & & \\
\hline Prbigo Bajo & 68,1 & 69,0 & 102 & 106 & 133,7 & 142,8 & 175,2 & 177,8 & 209,1 & 211,8 & 227,9 & 244,6 & & & & \\
\hline
\end{tabular}

Tabla 2.-Longitudes retrocalculadas $\left(\mathrm{L}\right.$, , nim.) y longitudes teóricas modelo de Von Bertalanffy $\left(\mathrm{L}^{\prime}\right.$, , mm.) a la edad $t$ de poblaciones Ch. polylepsis en varios ríos de la Cuenca del Duero.

Length back-calculated $\left(\mathrm{L},, \mathrm{mm}\right.$.) and theoretical length $\left(\mathrm{L}^{\prime}, \mathrm{mm}\right)$ von Bertalanffy's model. for age of ( $h$. poly/ $p$ sis from rivers of Duero Basin.

llo verdadero se forma aproxiniadamente a los 16 meses de vida (Sostoa y Sostoa, 1981).

Los modelos de crecimiento utilizados son los debidos a Von Rertalanffy (1938) cuya ecuación de crecimiento lineal para una longitud $\left(\mathrm{L}_{1}\right)$ a la edad $t$ es: $\mathrm{L}_{1}$ $=\mathrm{L}_{\infty}\left(1-\mathrm{e}^{-\mathrm{k}(\mathrm{t}-\mathrm{to})}\right)$. Donde, $\mathrm{L}_{\mathrm{x} \text { e }}$ es la asintota de la curva de crecimiento; $k$ es la tasa de crecimiento; $t$ es el tiempo equivalente al momento hipotético en el que la longitud del pez es 0 ; y $E$ (tasa de anabolis) es igual a k.L.

$L$ se estimó a partir del modelo gráfico de Ford
(1933) y Walford (1947). La tasa de crecimiento $k$ y $t_{\text {" }}$ se estimaron por el método de Dickie (1978).

Análogamente la ecuación de crecimiento en peso es: $P=P \quad\left(1-\mathrm{e}^{-k(1-t} \mathrm{o}\right)^{h}$.

La relación longitud-peso viene dada por la ecuación: $\mathrm{P}=\mathrm{a} \cdot \mathrm{L}^{\mathrm{h}}$, donde $b$ es un exponente próximo a 3, y $a$ es el coeficiente mórfico. Esta ecuación se obtiene a partir de una transformación logarítmica de la mis$\mathrm{ma}, \log \mathrm{P}=\log \mathrm{a}+\mathrm{b} \log \mathrm{L}$. hallando la recta de regresión por mínimos cuadrados.

Para calcular el coeficiente mórfico (a') de cada po- 


$\begin{array}{lcccccccc}\text { RIO } & \mathrm{K} & \text { pte. } & \text { Alcalin. } & \text { C'onduc. } & \text { Dureza } & \text { Fosfatos } & \text { Nitratos } & \text { T estv. } \\ \text { Duero } & 0,14 & 1,3 & 105.2 & 282,6 & 142.9 & 0,53 & 1,02 & 21,8 \\ \text { Arlanza } & 0,16 & 2,0 & 135.0 & 2975 & 147.5 & 0,30 & 0,53 & 22,0 \\ \text { Esgueva } & 0,26 & & 219.0 & 400.0 & 235,0 & 0,29 & 1.93 & 16,5 \\ \text { Carrión } & 0,06 & 3,2 & 67.0 & 139.0 & 73.5 & 0,28 & 0,83 & 21,0 \\ \text { Pisuerga } & 0,18 & 1,0 & 134.5 & 350.0 & 190.0 & 0,48 & 0,85 & 23,0 \\ \text { Esla } & 0.03 & 1,7 & 104,2 & 213.5 & 106,7 & 0.45 & 0.77 & 22,3 \\ \text { Orbigo Bajo } & 0,03 & 1,6 & 88,0 & 183.1 & 90.0 & 0.24 & 0.84 & 23,5\end{array}$

Tabla 3.-Tasa de crecimiento $(\mathrm{K})$ de (h. polylepis y valores medios de los factores físico-químicos del agua: alcalinidad (mg/1). conductividad ( $\mathrm{uS} / \mathrm{cm}$.), dureza (mg/1), fosfatos (mg/1), nitratos $(\mathrm{mg} / 1)$, temperatura media estival ( ${ }^{\circ} \mathrm{C}$.) y pendiente $(\%)$, en distintos ríos de la Cuenca del Duero.

Growth rates $(\mathrm{K})$ of $c h$ polylepis and mean values ofwnter physical-chemical factors in diferents rivers ofthe Duero Basin.

blación hemos supuesto un crecimiento isométrico (b = 3) y hemos ajustado la relación longitud-peso a una cúbica: $\mathrm{P}=$ a. $\mathrm{L}^{3}$. En este caso se consideraron también los ríos Ucero (Uc), alto Orbigo (Or-a) y alto Tormes (To-a). ya que disponíamos de datos suficientes para hallar sus coeficientes mórficos.

Utilizando la relación longitud del cuerpo/radio de la escama y el valor de los radios a cada annulus, se calcularon las longitudes retrocalculadas. La relación de la línea de regresión: $L m=a+b . R$, es representada por la ecuación modificada de la misma (Fraser y Lee, 1920): $\mathrm{L},-\mathrm{a}=\mathrm{R}_{\mathrm{t}} / \mathrm{R}_{\mathrm{n}}\left(\mathrm{L}_{\mathrm{n}}-\mathrm{a}\right)$, donde $L_{t}=$ longitud a la edad $t: \mathrm{R}=$ radio de la escama en el annulur. $t: \mathbf{L}_{n}=$ longitud del ejemplar; $\mathbf{R}_{\mathrm{n}}=$ radio de la escama: y a es un factor de conversión.

Para conocer la influencia de los factores físicoquímicos del agua en el crecimiento, se relacionó la tasa de crecimiento $(\mathrm{K})$ con: la alcalinidad. conductividad, dureza, fosfatos, nitratos, temperatura media estival y pendiente del río. La significación de la regresión se hizo con una t-Student (Parker, 1976).

\section{RESULTADOS \\ Edad}

La edad máxima encontrada en toda la Cuenca ha sido la clase de edad VIII, aunque aparece en una proporción muy pequeña $(<2 \%)$ con respecto al total de todos los ejemplares. Sin embargo Pérez (1979) encuentra edades de hasta 10 años en poblaciones de bogas del río Esla.

\section{Crecimiento lineal}

Los valores de los parámetros de crecimiento de Von Bertalanffy aparecen en la tabla 1. La tasa de crecimiento $(\mathrm{K})$ se encuentra entre el límite mínimo del río Esla, 0,03, y el límite máximo 0,26 del río Esgucva. Las ecuaciones de crecimiento lineal aparecen representadas en la figura $1-\mathrm{A}$, y las longitudes teóricas obtenidas de dichas ecuaciones para cada clase. se hallan cn la tabla 2.

\section{Relación longitud-peso}

La relación longitud-peso es de tipo exponencial y viene definida por la ecuación: $P=a \cdot L^{h}(P, g r ; ~ L$, $\mathrm{mm}$.). Los resultados obtenidos son:

$$
\begin{aligned}
& \text { Du-a: } P=1,33.10^{-5} \times \mathrm{L}^{2.99} \\
& \text { Du-b: } P=8,55 \cdot 10^{-6} \times \mathrm{L}^{3.09} \\
& \text { Or-b: } P=5,12.10^{-6} \times \mathrm{L}^{3.18} \\
& \text { Pi: } \mathrm{P}=1,84.10^{-5} \times \mathrm{L}^{2,94} \\
& \text { Aa: } \mathrm{P}=3,19 \cdot 10^{-5} \times \mathrm{L}^{2.81} \\
& \text { Eg: } \mathrm{P}=1,63 \cdot 10^{-5} \times \mathrm{L}^{2,97} \\
& \text { Ca: } P=2.12 .10^{-6} \times \mathrm{L}^{3,32} \\
& \text { El: } P=1,27.10^{-6} \times \mathrm{L}^{3,46}
\end{aligned}
$$

Cuando hacemos $b=3$, los valores de $a^{\prime}\left(\mathrm{gr} / \mathrm{mm}^{3}\right)$ resultantes de la ecuación: $P=a^{\prime} . L^{3}$, son ordenados de mayor a menor, y considerando en este caso los ríos Ucero y Tormes: 1) Eg: $\left.1,41.10^{-5} ; 2\right) \mathrm{Du}-\mathrm{b}$ : $\left.1,40.10^{-5}: 3\right)$ Pi: $1,38 \cdot 10^{-5}$; 4) Or-b: $1,33 \cdot 10^{-5}$; 5) El: $1,32 \cdot 10^{-5}$; 6) Du-a: $1,26 \cdot 10^{-5}$; 7) Uc: $\left.1,22 \cdot 10^{-5}: 8\right)$ Or-a: $\left.\quad 1,21 \cdot 10^{-5} ; 9\right)$ To-a: $\left.\quad 1,21 \cdot 10^{-5} ; 10\right) \mathrm{Ca}$ : $\left.1,20 \cdot 10^{-5} ; 11\right)$ Aa: $1,20 \cdot 10^{-5}$.

Se vio que existía correlación significativa entre los coeficientes mórficos (a') obtenidos en las ecuaciones anteriores y las pendientes de los ríos. El resultado de la recta de regresión y el test de significación de la misma (Parker, 1976), fue:

$\mathrm{a}^{\prime}=1,33-0.061 \log \mathrm{i}$ (siendo $\mathrm{i}$ la pendiente en $\%$ ) $\mathrm{r}=0,81(\mathrm{t}=3,89 ;$ g.l. $=8 ; \mathrm{P}<0,02)$ 2-H.

La recta de regresión resultante aparece en la figura

\section{Crecimiento en peso}

Los valores de parámetro $\mathrm{P}(\mathrm{gr})$ para cada río son los siguientes: Du-a: 369,292; Du-b: 511,110; Pi: 489,350; Or-b: 34.275,174; El: 49.497,120; Ca: 4.154.820: Eg: 667.35; Aa: 559,96. Las ecuaciones de crecimiento en peso se representan en la figura 1-B.

\section{Retrocalculo}

Las ecuacioncs retrocalculadas obtenidas son:

Du-a: $L_{1}=35,26+\mathrm{R} / \mathrm{R} \quad(\mathrm{L},-35,26)$

$\mathrm{Du}-\mathrm{b}: \mathrm{L}_{1}^{\prime}=27,37+\mathrm{R}^{\prime} / \mathrm{R}^{n}\left(\mathrm{~L}_{\mathrm{n}}-27,37\right)$

Pi: $L=40,53+R / R\left(L^{\prime}-40.53\right)$

Aa: $L_{1}=31,38+R_{1} / R_{n}\left(L_{n}^{n}-31,38\right)$ 

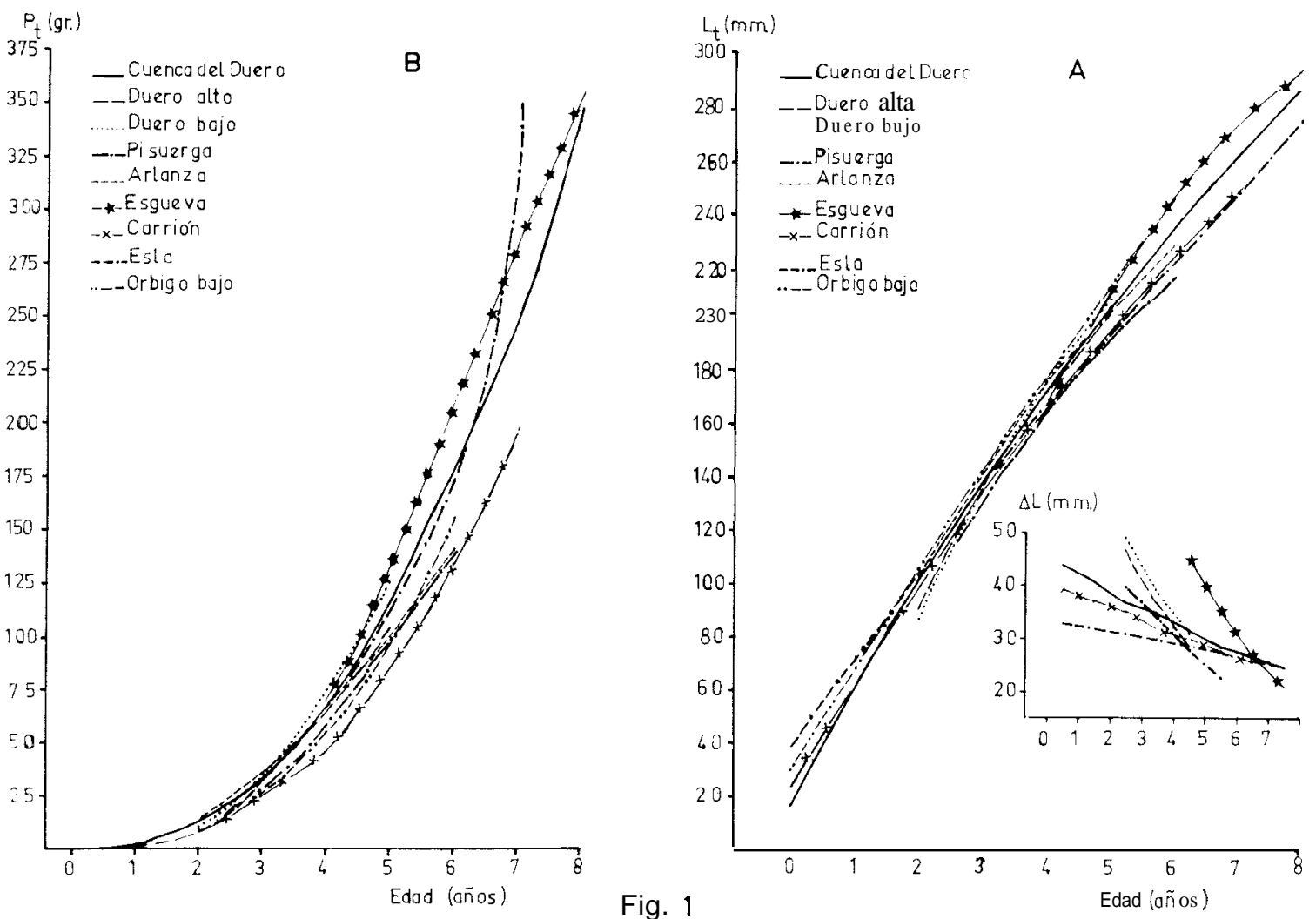

Figura 1-A.-Representación gráfica de los valores teóricos de longitud $\left(\mathrm{L}_{\mathrm{t}}\right)$, modelo de Von Bertalanfiy. 1938. e incrementos anuales en longitud de Ch.polylepsis según la edad en varios ríos de la Cuenca del Duero.

Plots of theoretical length (von Bertalanffy's model. 1938) and increase annual in length and ages of Ch. polylepsis in some spanish rivers.

Figura 1-B.-Representación gráfica de los valores teóricos de peso (P,). modelo de Von Bertalanfly, 1938. de c $h$ polyle’psis según la edad en varios rios de la Cuenca del Duero.

Plots oftheoretical weight (von Bertalanffy"s model, 1938)and ages of $(\%$. polykpsis in some spanish rivers.

Eg: $L_{t}=69,65+R / R_{n}\left(L_{n}-69,65\right)$

Ca: $L_{1}=31,33+R^{t} / R^{n}\left(L_{n}-31,33\right)$

El: $L_{t}=18,83+R_{t} / R_{n}\left(L_{n}-18,83\right)$

Or-b: $L_{1}=29.37+R_{1} / R_{n}\left(L_{n}-29.37\right)$

En la tabla 2 aparecen las longitudes medias retrocalculadas para cada edad y para cada río.

\section{DISCUSION}

Las poblaciones de bogas de la Cuenca del Duero que presentan mayores tasas de crecimiento longitudinal $(\mathrm{K})$ coincidiendo con los valores máximos de tasa de anabolismo (E) pertenecen a los tramos alto y bajo del río Duero y a los ríos Esgueva. Arlanza y Pisuerga. Por otra parte, Lobon-Cervia y Elvira (1981) y Lobon-Cervia (1982) obtienen tasas de anabolismo en bogas del embalse de Pinilla y del río Jarama respectivamente dentro del intervalo de valores encontrados por nosotros (tabla 1). Sin embargo, Pérez (1979) obtiene tasas de crecimiento y de anabolismo en el río Esla superiores posiblemente debido a que las poblaciones estudiadas por nosotros estaban padeciendo un periodo largo de sequía.

Las bogas de los ríos: Eg, Du-b. Pi. Or-b y El. son las que presentan coeficientes mórficos mayores y por tanto con formas más redondeadas, estos coeficientes mórficos, también llamados factor de condición $(\mathrm{K}=$ $\mathrm{P} / \mathrm{L}^{3}$ ), son semejantes a los hallados por Eiras y Guoveia (1979) en los ríos portugueses Támega y Sousa y superiores a los encontrados en el río Ferreira por el mismo autor y en el Jarama por Lobon-Cervia (1982): sin embargo. son inferiores al encontrado por Lobon-Cervia y Elvira (1981) en el cmbalse de Pini- 

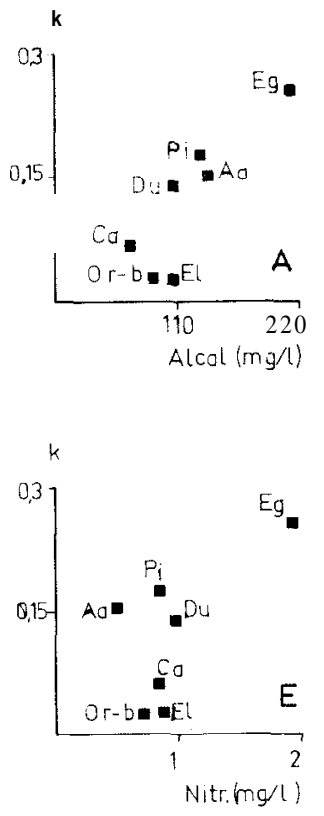
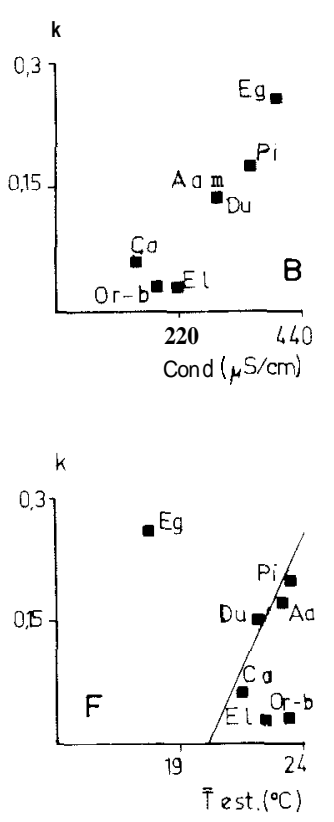
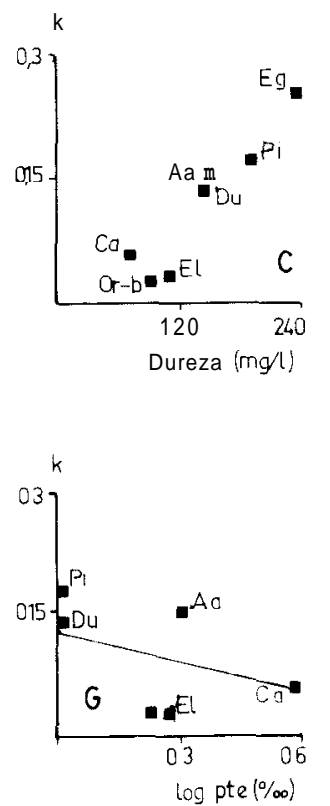
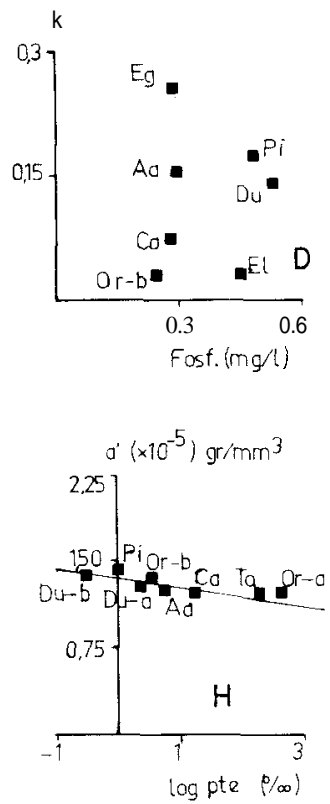

Fig. 2

Figura 2.-Relación de la tasa de crecimiento (K) y coeficiente mórfico (a') de la especie (h.polylepsis en los ríos Carrión (Ca), Arlanza (Aa), Esgueva (Eg). Duero (Du), Orbigo alto (Or-a), Tormes (To). Orbigo bajo (Or-b),Pisuerga (Pi)y Esla (El), con: (A)alcalinidad. (mg/1), (B)conductividad (S/cm.), (C) dureza (mg/1), (D)fosfatos (mg/1), (E) nitratos (mg/1), (F) temperatura media estival ("C.), (G)y (H) pendiente del río ( $\%$ oo $)$

Relationship between growth in length rates and morphic coeffcient of $C h$ polylepsis in rivers ofthe Duero Basin with: (A)alkalinity, (B) conductivity, (C) $\mathrm{CO}_{3} \mathrm{Ca},(\mathrm{D})$ phosphate, (E) nitrate, $(\mathrm{F})$ water mean summer temperature, $(\mathrm{G})$ y $(\mathrm{H})$ slope of river.

lla, lo que permite mantener la idea de que los embalses son hábitats realmente favorables para el crecimiento de las bogas.

\section{Factores ambientales que controlan el crecimiento}

Para analizar las condiciones mesológicas que catacterizan los crecimientos de las poblaciones de bogas estudiadas en la Cuenca del Duero, tomamos los datos mesológicos de González del Tánago y García de Jalón (1983)

De los resultados obtenidos de los coeficientes mórficos se puede deducir que, en general, las bogas de los tramos altos de los ríos (Du-a y Or-a) son más estilizadas que las presentes en los tramos bajos $(\mathrm{Du}-\mathrm{b}$ y Or-b).

Tanto el coeficiente mórfico como la tasa de crecimiento están en relación inversa con la pendiente del rio (figura $2 \mathrm{G}-\mathrm{H}$ ). La boga se encuentra en pendientes que van de 0,3 a $6 \%$ (García de Jalón y López Alvarez, 1981); por encima de lo cual no aparecen a excepción de los tramos altos de los ríos Pisuerga
$(15 \%$ de pendiente) del río Orbigo $(13 \%)$ del Tormes $(10 \% /(x)$ y del Agueda $(12 \% /(0))$. Esto puede ser debido a la presencia de embalses en las proximidades, aguas abajo de dichas estaciones, permitiendo a la boga refugiarse durante las épocas de crecida en las colas de los embalses.

Los resultados obtenidos del estudio mesológico de la boga (tabla 3) demustran que, generalmente, las poblaciones de boga con tasas de crecimiento normales, alrededor de 0,14 (Duero, Pisuerga, Esgueva y Arlanza), se encuentran en un medio que al menos cumple 2 de las 3 condiciones siguientes, sino las tres como es el caso de Du y del Pi; elevada mineralización de las aguas, estimada a partir de la conductividad (> $220 \mathrm{uS} / \mathrm{cm}$ ), la alcalinidad ( $>105 \mathrm{mg} / 1$ ) y la dureza $(>120 \mathrm{mg} / 1$ ) (figura 2-A, 2-B, 2-C); alto contenido en nutrientes reflejado en fosfatos $(>0,3$ $\mathrm{mg} / 1$ ) y nitratos (>0,85 mg/1) (figura 2-D, 2-E); y con temperaturas medias estivales relativamente altas, superiores a $22^{\circ} \mathrm{C}$. (figura $2-\mathrm{F}$ ). Ello es razonable ya que estas tres condiciones son también factores 
limitantes del crecimiento de algas y macrófitas que constituyen la alimentación básica de la boga (Kacanski. el ul., 1980).

De esta forma, las poblaciones con tasas de crecimiento menores a lo normal, como es el caso de las pertenecientes a Or-b. El y Ca, aparecen en una situación de escasa mineralización y bajo contenido en nutrientes fundamentalmente además de encontrarse con temperaturas medias estivales relativamente bajas (como es el caso del Carrión). Los bajos crecimientos que se dan en las poblaciones del Or-b y El son debidos al escaso contenido en nutrientes y principalmente a la baja mineralización de sus aguas.

\section{BIBLIOGRAFIA}

Dickie, L.M. 1978. Mathematical models of Growth In: Methods for assessment of fish production in fresh waters (Bagenal, T. ed.): 130-136. 1.B.P. handbook 3 Oxford. $365 \mathrm{pp}$.

Eiras, J.; Gouveia, A. 1979. Note sur I'influence du mileiu dans la rélation poids-longueur et facteur de condition dans trois sous-especes de poissons du nord de Portugal. Puhl. Inst. Zoo. "Dr. Augusto Nobre». 144: 11-20.

Ford, E. 1933. An account of the Herring investigations conducted at Plymouth during the years 1924-1933. J. mur. Biol. Ass. U. K., 19: 305-384.

Fraser, C.Mc.L. 1916. Growth of the spring salmon. Trans Pacif. Fish Soc. Seattle. Second ann. Meting. 1915: 29-35.

García de Jalón, D.; López Alvarez, J.V. 1981. Distribución geográfica y mesológica de las especies piscicolas de la cuenca del Duero en el verano de 1981 Actas del Primer Congreso Español de Limnologia. 227-235.

García de Jalón, D.; González del Tánago, M. 1983. Estudio biotipológico de las comunidades piscícolas de la cuenca del Duero. Bol. Est. Centr. Ecol. 12(24): 57-66.

González del Tánago, M.; García Jalón, D. 1983. Estudio para una metodología de clasificación biotipológica de los ríos españoles según el modelo propuesto por la C.E.E.: Aplicación a la cuenca del Duero (CEOTMA. MOPU. Madrid) (en prensa).

Granado, L.; García-Novo, F. 1983. Biología y crecimiento de la boga de río. Chondrostoma polylepis (Steind., 1865). VI Reun. Bie. de la R. Soc. Esp. His. Nat., Santiago, 1983.

Kacanski, D.; Hafner, D.; Blagojevic, S.; Kosoríc, D. 1380. On the nutrition of some cyprinid fish species from the Bosna river. Icthvologia 13(1): 51-61.

Laevastu, T. 1980. Mediciones de longitud de peces. In: Mamual de métodos de biología pesquera (Laevastu, T. ed.): 196-202. Acribia, Zaragoza, 243 pp.

Lobon-Cervia, J.; Elvira, B. 1981. Edad, crecimiento y reproducción de la boga de río (Chondrostoma polylepis Steind., 1865) en el embalse de Pinilla (río Lozoya). Bol. Inst. Esp. Oceano. 6: 200-213.

Lobon-Cervia, J. 1982. Population analysis of the lberian Nase (Chondostoma polylepis Stein., 1865) in the Jarama river. Vie Milieu. 32(3): 139-148.

Parker, K.C. 1976. Estadistica para biólogos. Omega, Barcelona, 136 pp.

Pérez, L. 1979. Catálogo íctico y estudio del crecimiento de tres ciprínidos del río Esla: sectores de montaña y meseta. Leon. Memoria de Licenciatura. Universidad de Leon

Sostoa, A.; Sostoa, F. 1981. Edad y crecimiento de Ch. toxostoma Vallot, 1937 en el curso inferior del río Ebro (Tarragona, España). Aclas del Primer Congreso Español de Limnologia

Von Rertalanffy, L. 1938. A yuantitative theory of organic growth. Hum. Biol. 10, 181-243.

Walford, L.A. 1947. A new graphic method of describing the growth of animals. Biol. Bull. mar. biol. Lab., Woods Holc., 90. 141-147. 